\title{
Two-phonon infrared absorption spectra of germanium and silicon calculated from first principles
}

\author{
G. Deinzer and D. Strauch \\ Institut für theoretische Physik, Universität Regensburg, D-93040 Regensburg, Germany \\ (Received 15 August 2003; published 26 January 2004)
}

\begin{abstract}
The two-phonon infrared absorption spectrum of the covalent elemental semiconductors $\mathrm{Si}$ and $\mathrm{Ge}$ is calculated completely $a b$ initio. Besides the harmonic phonon eigen solutions this involves the phonon-photon coupling constants, the so called second-order dipole moments (also called dipole coefficients). These are given by the third-order derivatives of the total energy with respect to an electric field (once) and to atomic displacements (twice). In the framework of density functional theory, we have applied the $2 n+1$ theorem to derive an analytic expression for the second-order dipole moments. Numerical calculations of these coefficients and of the infrared absorption spectrum of Si and Ge are carried out. The shape and overall intensities of the spectra compare well with experimental data, even though some discrepancies remain.
\end{abstract}

DOI: 10.1103/PhysRevB.69.045205

PACS number(s): 78.30.Am, 31.15.Ar, 71.15.Mb

\section{INTRODUCTION}

In recent years, density functional theory (DFT) has been proven to be a powerful tool to determine both the groundstate and the linear-response properties of materials. Within the linear-response theory in the framework of DFT, i.e., the so called density-functional perturbation theory (DFPT), phonon frequencies and eigenvectors have been calculated in excellent agreement with experimental data. ${ }^{1,2}$

The extension to the third order via the so-called $2 n+1$ theorem $^{3}$ also makes the nonlinear regime accessible. ${ }^{4}$ Within this approach anharmonic force constants, ${ }^{5-10}$ nonlinear susceptibilities, ${ }^{11,12}$ and Raman tensors ${ }^{13}$ have been calculated.

In this work we investigate a further nonlinear property of materials: Due to the inversion symmetry of the elemental covalent semiconductors one-phonon infrared (IR) absorption is forbidden, and the absorption is dominated by twophonon processes, the photon-phonon coupling constants being the second-order dipole moments. The shape of the twophonon infrared absorption spectrum has two ingredients: (a) harmonic phonon eigenfrequencies and eigenvectors and (b) second-order dipole moments.

First attempts to calculate the infrared absorption in these materials have been performed by Kress et al. ${ }^{14}$ using (a) harmonic phonon properties from a shell model with phenomenological input for the model parameters as well as (b) model parameters for the second-order dipole moments.

The second-order dipole moments can be regarded as the change of the effective charges with respect to atomic displacements. Ab-initio calculations with harmonic-phonon properties from response theory and second-order dipole moments from frozen-phonon-like derivatives of the effective charges have been presented by Strauch and co-workers. ${ }^{15,16}$ Here the coefficients had been incomplete: They had been restricted to only second neighbors ${ }^{15}$ and some coefficients had been left undetermined (due to an incomplete set of superlattices necessary in the frozen-phonon method) and, in order to minimize the arbitrariness, had been set equal to zero, ${ }^{16}$ also see Sec. IV.
In this paper we will derive an analytic expression for the third-rank tensor of the second-order dipole moments in the framework of nonlinear DFPT. To remedy the shortcomings of the previous theoretical work we will quantitatively calculate a complete set of the tensor elements, which describe the interaction up to eighth neighbors within DFPT and from these (and from the harmonic eigen solutions) the absorption spectrum.

\section{SECOND-ORDER DIPOLE MOMENTS}

The part of the Hamiltonian which describes the interaction of the crystal with an external electric field $\mathbf{E}$ is

$$
H^{\prime}=-\sum_{\alpha} M_{\alpha} E_{\alpha} .
$$

$\mathbf{M}$ is the electrical dipole moment of the crystal which can be expanded in terms of atomic displacements $u_{\alpha}^{\kappa}(\mathbf{R})$, where $\alpha$ is the cartesian index and $\kappa$ labels the atom in the unit cell at lattice vector $\mathbf{R}$,

$$
\begin{aligned}
M_{\alpha}= & M_{\alpha}^{0}+\sum_{n \geqslant 1} \frac{1}{n !} \sum_{\substack{\mathbf{R}_{1} \ldots \mathbf{R}_{n} \alpha_{1} \\
\kappa_{1} \ldots \kappa_{n}}} \sum \\
& \times M_{\alpha, \alpha_{1} \ldots \alpha_{n}}^{\kappa_{1} \ldots \kappa_{n}}\left(\mathbf{R}_{1}, \ldots, \mathbf{R}_{n}\right) u_{\alpha_{1}}^{\kappa_{1}}\left(\mathbf{R}_{1}\right) \cdots u_{\alpha_{n}}^{\kappa_{n}}\left(\mathbf{R}_{n}\right) .
\end{aligned}
$$

The expansion coefficients are the static polarization $M_{\alpha}^{0}$, the Born effective charges $M_{\alpha, \alpha_{1}}^{\kappa_{1}}$, the second-order dipole moments $M_{\alpha, \alpha_{1} \alpha_{2}}^{\kappa_{1} \kappa_{2}}$, etc. The calculation of the polarization $M_{\alpha}^{0}$ can be performed by Berry-phase techniques. ${ }^{17,18}$ The Born effective charges $M_{\alpha, \alpha_{1}}^{\kappa_{1}}$ are accessible within standard linear DFPT. ${ }^{1}$ The second-order dipole moments are defined by the third-order derivative

$$
M_{\alpha, \alpha_{1} \alpha_{2}}^{\kappa_{1} \kappa_{2}}\left(\mathbf{R}_{1}, \mathbf{R}_{2}\right)=-\frac{\partial^{3} \mathcal{E}}{\partial E_{\alpha} \partial u_{\alpha_{1}}^{\kappa_{1}}\left(\mathbf{R}_{1}\right) \partial u_{\alpha_{2}}^{\kappa_{2}}\left(\mathbf{R}_{2}\right)}
$$


of the total energy $\mathcal{E}$. For evaluating this quantity, one introduces the Fourier transform

$$
M_{\alpha, \alpha_{1} \alpha_{2}}^{\kappa_{1} \kappa_{2}}(\mathbf{q})=\sum_{\mathbf{R}_{1}, \mathbf{R}_{2}} M_{\alpha, \alpha_{1} \alpha_{2}}^{\kappa_{1} \kappa_{2}}\left(\mathbf{R}_{1}, \mathbf{R}_{2}\right) \mathrm{e}^{\mathrm{i} \mathbf{q} \cdot\left(\mathbf{R}_{1}-\mathbf{R}_{2}\right)}
$$

The elements of this tensor can be evaluated in DFPT using the $2 n+1$ theorem. ${ }^{3}$ This theorem states that the knowledge of the Kohn-Sham wave functions and Hamiltonians (except the external potential) up to order $n$ is sufficient for the calculation of response functions up to order $2 n+1$. In practical calculations, not all of the perturbed wavefunctions but just the part projected on the unoccupied states are calculated. ${ }^{1}$ To derive an analytic expression for the second-order dipole coefficients, we follow the general description given by Debernardi and Baroni. ${ }^{4}$ For the convenience of the reader we repeat their final result for the thirdorder change in energy with respect to perturbations $\lambda_{i}(i$ $=1,2,3)$,

$$
\frac{\partial^{3} \mathcal{E}}{\partial \lambda_{1} \partial \lambda_{2} \partial \lambda_{3}}=\sum_{\text {perm }} \widetilde{E}^{\lambda_{1} \lambda_{2} \lambda_{3}}
$$

where the sum is performed over all six permutations of the perturbations $\lambda_{i}$, and where

$$
\begin{aligned}
\widetilde{E}^{\lambda_{1} \lambda_{2} \lambda_{3}}= & \sum_{v}\left(\left\langle\psi_{v}^{\lambda_{1}}\left|\mathcal{P}_{c} v_{\mathrm{KS}}^{\lambda_{2}} \mathcal{P}_{c}\right| \psi_{v}^{\lambda_{3}}\right\rangle+\left\langle\psi_{v}^{\lambda_{1}}\left|\mathcal{P}_{c} v_{\mathrm{ext}}^{\lambda_{2} \lambda_{3}}\right| \psi_{v}^{0}\right\rangle\right. \\
& \left.+\left\langle\psi_{v}^{0}\left|v_{\mathrm{ext}}^{\lambda_{1} \lambda_{2}} \mathcal{P}_{c}\right| \psi_{v}^{\lambda_{3}}\right\rangle+\left\langle\psi_{v}^{0}\left|v_{\mathrm{ext}}^{\lambda_{1} \lambda_{2} \lambda_{3}}\right| \psi_{v}^{0}\right\rangle\right) \\
& -\sum_{v v^{\prime}}\left\langle\psi_{v}^{\lambda_{1}}\left|\mathcal{P}_{c}\right| \psi_{v^{\prime}}^{\lambda_{2}}\right\rangle\left\langle\psi_{v^{\prime}}^{0}\left|v_{\mathrm{KS}}^{\lambda_{3}}\right| \psi_{v}^{0}\right\rangle \\
& +\frac{1}{6} \int \mathrm{d}^{3} r \mathrm{~d}^{3} r^{\prime} \mathrm{d}^{3} r^{\prime \prime} K^{3}\left(\mathbf{r}, \mathbf{r}^{\prime}, \mathbf{r}^{\prime \prime}\right) \\
& \times n^{\lambda_{1}}(\mathbf{r}) n^{\lambda_{2}}\left(\mathbf{r}^{\prime}\right) n^{\lambda_{3}}\left(\mathbf{r}^{\prime \prime}\right) .
\end{aligned}
$$

Here, the superscript $\lambda_{i}$ denotes the derivative with respect to the perturbation $\lambda_{i}$, and the derivatives have to be taken at the unperturbed ground-state density. The sums run over the unperturbed (occupied) valence-band states;

$$
v_{\mathrm{KS}}=v_{\mathrm{ext}}+v_{\mathrm{Hxc}}
$$

is the Kohn-Sham potential with $v_{\text {ext }}$ the external potential and $v_{\mathrm{Hxc}}$ the Hartree, exchange, and correlation potential;

$$
\mathcal{P}_{c}=1-\sum\left|\psi_{v}^{0}\right\rangle\left\langle\psi_{v}^{0}\right|
$$

is the projector onto the subspace of unperturbed conduction states; and

$$
K^{3}\left(\mathbf{r}, \mathbf{r}^{\prime}, \mathbf{r}^{\prime \prime}\right)=\frac{\partial^{3} E_{\mathrm{xc}}[n]}{\partial n(\mathbf{r}) \partial n\left(\mathbf{r}^{\prime}\right) \partial n\left(\mathbf{r}^{\prime \prime}\right)} .
$$

In Eq. (4) the result of the $2 n+1$ theorem is demonstrated and to be noted: Only the external potential $v_{\text {ext }}$ is needed in second and third order, while the wave functions and the Hartree, exchange, and correlation potential $v_{\mathrm{Hxc}}$ are needed only up to first order. In the framework of the linear-response theory, the derivatives are calculated self consistently via the so-called Sternheimer equation. ${ }^{1}$

In the following we use the periodic part

$$
u_{v \mathbf{k}}(\mathbf{r})=\psi_{v \mathbf{k}}(\mathbf{r}) \mathrm{e}^{-\mathrm{ik} \cdot \mathbf{r}}
$$

of the Kohn-Sham wave function $\psi_{v \mathbf{k}}$ with valence band in$\operatorname{dex} v$ and wave vector $\mathbf{k}$, and the projector

$$
\mathcal{P}_{c}=1-\sum\left|u_{v \mathbf{k}}\right\rangle\left\langle u_{v \mathbf{k}}\right|
$$

For the derivative with respect to an electric field one needs a representation of the position operator $\mathbf{r}$ in the basis of Bloch orbitals, which appears in the first-order Hamiltonian. It can be shown ${ }^{19}$ that the position operator $\mathbf{r}$ transforms into the derivative with respect to the $\mathbf{k}$ vector,

$$
\mathbf{r} \psi_{v \mathbf{k}}(\mathbf{r})=-\mathrm{i} \nabla_{\mathbf{k}} \psi_{v \mathbf{k}}(\mathbf{r})+\mathrm{e}^{\mathrm{i} \mathbf{k} \cdot \mathbf{r}_{\mathrm{i}}} \nabla_{\mathbf{k}} u_{v \mathbf{k}}(\mathbf{r})
$$

Substituting the present perturbations as in Eq. (1) for the general perturbations as in Eq. (3) we obtain

$$
M_{\alpha, \alpha^{\prime} \alpha^{\prime \prime}}^{\kappa^{\prime} \kappa^{\prime \prime}}(\mathbf{q})=\tilde{M}_{\alpha, \alpha^{\prime} \alpha^{\prime \prime}}^{\kappa^{\prime} \kappa^{\prime \prime}}(\mathbf{q})+\tilde{M}_{\alpha, \alpha^{\prime \prime} \alpha^{\prime}}^{\kappa^{\prime \prime} \kappa^{\prime}}(\mathbf{q}),
$$

where

$$
\begin{aligned}
& \frac{1}{\Omega} \tilde{M}_{\alpha, \alpha^{\prime} \alpha^{\prime \prime}}^{\kappa^{\prime} \kappa^{\prime \prime}}(\mathbf{q})=\sum_{v} \int_{\mathrm{BZ}} \frac{\mathrm{d}^{3} k}{(2 \pi)^{3}}\left(4\left\langle\frac{\partial u_{v \mathbf{k}}}{\partial E_{\alpha}}\left|\mathcal{P}_{c} \frac{\partial v_{\mathrm{KS}}}{\partial u_{\alpha^{\prime}}^{\kappa^{\prime}(-\mathbf{q})}} \mathcal{P}_{c}\right| \frac{\partial u_{v \mathbf{k}}}{\partial u_{\alpha^{\prime \prime}}^{\kappa^{\prime \prime}(\mathbf{q})}}\right\rangle+2\left\langle\frac{\partial u_{v \mathbf{k}}}{\partial u_{\alpha^{\prime}}^{\kappa^{\prime}(\mathbf{q})}}\left|\mathcal{P}_{c} \frac{\partial v_{\mathrm{Hxc}}}{\partial E_{\alpha}} \mathcal{P}_{c}\right| \frac{\partial u_{v \mathbf{k}}}{\partial u_{\alpha^{\prime \prime}}^{\kappa^{\prime \prime}}(\mathbf{q})}\right\rangle\right) \\
& +\delta_{\kappa^{\prime}, \kappa^{\prime \prime}} 4 \sum_{v} \int_{\mathrm{BZ}} \frac{\mathrm{d}^{3} k}{(2 \pi)^{3}}\left\langle\frac{\partial u_{v \mathbf{k}}}{\partial E_{\alpha}}\left|\mathcal{P}_{c} \frac{\partial^{2} v_{\mathrm{ext}}}{\partial u_{\alpha^{\prime}}^{\kappa^{\prime}}(\mathbf{q}=0) \partial u_{\alpha^{\prime \prime}}^{\kappa^{\prime}}(\mathbf{q}=0)}\right| u_{v \mathbf{k}}^{(0)}\right\rangle \\
& -\sum_{v v^{\prime}} \int_{\mathrm{BZ}} \frac{\mathrm{d}^{3} k}{(2 \pi)^{3}}\left\langle 4\left\langle\frac{\partial u_{v \mathbf{k}}}{\partial E_{\alpha}}\left|\mathcal{P}_{c}\right| \frac{\partial u_{v^{\prime} \mathbf{k}+\mathbf{q}}}{\partial u_{\alpha^{\prime}}^{\kappa^{\prime}}(-\mathbf{q})}\right\rangle\left\langle u_{v^{\prime} \mathbf{k}+\mathbf{q}}^{(0)}\left|\frac{\partial v_{\mathrm{KS}}}{\partial u_{\alpha^{\prime \prime}}^{\kappa^{\prime \prime}}(\mathbf{q})}\right| u_{v \mathbf{k}}^{(0)}\right\rangle\right. \\
& \left.+2\left\langle\frac{\partial u_{v \mathbf{k}}}{\partial u_{\alpha^{\prime}}^{\kappa^{\prime}}(\mathbf{q})}\left|\mathcal{P}_{c}\right| \frac{\partial u_{v^{\prime} \mathbf{k}}}{\partial u_{\alpha^{\prime \prime}}^{\kappa^{\prime \prime}}(\mathbf{q})}\right\rangle\left\langle u_{v \mathbf{k}}^{(0)}\left|\frac{\partial v_{\mathrm{Hxc}}}{\partial E_{\alpha}}\right| u_{v \mathbf{k}}^{(0)}\right\rangle\right)
\end{aligned}
$$




$$
\begin{aligned}
& +2 \sum_{v v^{\prime}} \int_{\mathrm{BZ}} \frac{\mathrm{d}^{3} k}{(2 \pi)^{3}}\left\langle u_{v \mathbf{k}}|| \frac{i e \partial}{\partial k_{\alpha}}|| u_{v^{\prime} \mathbf{k}}\right\rangle\left\langle\frac{\partial u_{v^{\prime} \mathbf{k}}}{\partial u_{\alpha^{\prime}}^{\kappa^{\prime}}(\mathbf{q})}\left|\mathcal{P}_{c}\right| \mathcal{P}_{c} \mid \frac{\partial u_{v \mathbf{k}}}{\partial u_{\alpha^{\prime \prime}}^{\kappa^{\prime \prime}}(\mathbf{q})}\right\rangle \\
& +\frac{1}{2} \int \mathrm{d}^{3} r f_{\mathrm{xc}}^{\mathrm{LDA}}(\mathbf{r}) \frac{\partial n(\mathbf{r})}{\partial E_{\alpha}} \frac{\partial n(\mathbf{r})}{\partial u_{\alpha^{\prime}}^{\kappa^{\prime}}(-\mathbf{q})} \frac{\partial n(\mathbf{r})}{\partial u_{\alpha^{\prime \prime}}^{\kappa^{\prime \prime}}(\mathbf{q})}
\end{aligned}
$$

$\Omega$ is the volume of the unit cell. The third-order exchange and correlation functional in the local density approximation is given by

$$
f_{\mathrm{xc}}^{\mathrm{LDA}}(\mathbf{r})=\delta\left(\mathbf{r}-\mathbf{r}^{\prime}\right) \delta\left(\mathbf{r}-\mathbf{r}^{\prime \prime}\right) K^{3}\left(\mathbf{r}, \mathbf{r}^{\prime}, \mathbf{r}^{\prime \prime}\right) .
$$

\section{DIELECTRIC SUSCEPTIBILITY AND ABSORPTION CONSTANT}

The infrared absorption of a crystal can be expressed in terms of the dielectric susceptibility $\boldsymbol{\chi}$. This is connected to the induced polarization $\mathbf{P}$ by

$$
\mathbf{P}=\frac{1}{V}[\mathbf{M}(\mathbf{E})-\mathbf{M}(\mathbf{E}=0)]=\varepsilon_{0} \chi \mathbf{E},
$$

where $\varepsilon_{0}$ is the vacuum permittivity. For cubic systems the susceptibility is proportional the unit tensor, and here we restrict ourselves to two-phonon processes,

$$
\chi(\omega)=\frac{\hbar}{V \varepsilon_{0}} \chi_{\alpha \alpha}^{(2)}(\omega) .
$$

By expanding the polarization in terms phonon field operators for phonons with quantum number $\lambda=(\mathbf{q}, j)$ (with wave vector $\mathbf{q}$ and branch index $j$ ) one obtains the second-order dipole moments

$$
\begin{aligned}
M_{\alpha}^{(2)}\left(\lambda_{1}, \lambda_{2}\right)= & \frac{1}{2 N \sqrt{\omega_{\lambda_{1}} \omega_{\lambda_{2}}}} \\
& \times \sum_{\substack{\kappa_{1} \kappa_{2} \\
\alpha_{1} \alpha_{2}}} M_{\alpha, \alpha_{1} \alpha_{2}}^{\kappa_{1} \kappa_{2}}(\mathbf{q}) \frac{e_{\alpha_{1}}^{\kappa_{1}}\left(\lambda_{1}\right)}{\sqrt{m_{\kappa_{1}}}} \frac{e_{\alpha_{2}}^{\kappa_{2}}\left(\lambda_{2}\right)}{\sqrt{m_{\kappa_{2}}}}
\end{aligned}
$$

with $\lambda_{1}=\left(\mathbf{q}, j_{1}\right)$ and $\lambda_{2}=\left(-\mathbf{q}, j_{2}\right)$. Here, $\omega_{\lambda}$ is the phonon frequency, $\mathbf{e}^{\kappa}(\lambda)$ the eigenvector, $m_{\kappa}$ the mass of the atom $\kappa$, and $M_{\alpha, \alpha_{1} \alpha_{2}}^{\kappa_{1} \kappa_{2}}(\mathbf{q})$ as in Eq. (2).

The two-phonon contribution to the imaginary part of the dielectric susceptibility is then ${ }^{20}$

$$
\begin{aligned}
\operatorname{Im} \chi_{\alpha \alpha}^{(2)}(\omega)= & \frac{\pi}{2} \sum_{j_{1} j_{2}, \mathbf{q}}\left|M_{\alpha}^{(2)}\left(\lambda_{1}, \lambda_{2}\right)\right|^{2} \sum_{+,-} \delta\left(\omega_{\lambda_{1}} \pm \omega_{\lambda_{2}}-\omega\right) \\
& \times\left[\left(n_{\lambda_{2}}+\frac{1}{2}\right) \pm\left(n_{\lambda_{1}}+\frac{1}{2}\right)\right],
\end{aligned}
$$

and can be looked at as the two-phonon density of states (TDOS)

$$
D_{2}(\omega)=\sum_{+,-} D_{2}^{ \pm}(\omega), \quad D_{2}^{ \pm}(\omega)=\sum_{\lambda_{1} \lambda_{2}} \delta\left(\omega_{\lambda_{1}} \pm \omega_{\lambda_{2}}-\omega\right)
$$

weighted by $\left|M_{\alpha}^{(2)}\left(\lambda_{1}, \lambda_{2}\right)\right|^{2}$ and by combinations of BoseEinstein occupation numbers

$$
n_{\lambda}=\left(\mathrm{e}^{\hbar \omega_{\lambda} / k T}-1\right)^{-1},
$$

which make the absorption depend on temperature. The two different signs in Eq. (10) refer to the so-called summation and difference processes.

The real part of the susceptibility is then given by the Kramers-Kronig (Hilbert) transform of the imaginary part.

One now writes the dielectric function

$$
\varepsilon(\omega)=\varepsilon_{\infty}+\chi(\omega)
$$

in the infrared energy regime as the sum of the electronic contribution $\varepsilon_{\infty}$ and the phonon contribution $\chi(\omega)$. The absorption coefficient is then given by

$$
\alpha_{\omega}=\frac{2 \omega n^{\prime \prime}(\omega)}{c}
$$

where $c$ is the speed of light and $n^{\prime \prime}(\omega)$ the imaginary part of the refractive index $n(\omega)=\sqrt{\varepsilon(\omega)}$.

\section{TECHNICAL DETAILS}

The pseudopotentials are generated following the scheme proposed by von Barth and Car. ${ }^{21}$ For the exchange and correlation energy we have used the local density approximation (LDA), as calculated by Monte Carlo techniques by Ceperly and Alder $^{22}$ and interpolated by Perdew and Zunger. ${ }^{23}$ The combination of the LDA and of these pseudopotentials had resulted in phonon dispersion curves ${ }^{1}$ in excellent agreement with experiment (using the theoretical lattice constant).

The integration over the Brillouin zone in Eq. (7) is performed using the method of special points on an $8 \times 8 \times 8$ Monkhorst-Pack k-vector mesh ${ }^{24}$ and using a cut-off energy of 24 Ry. The derivative appearing in equation (7) with respect to $\mathbf{k}$ is evaluated by finite differences using the method of Marzari and Vanderbilt. ${ }^{25}$ To this end, ${ }^{26}$ additional points are constructed at a distance of $0.002 \sqrt{3}\left(2 \pi / a_{0}\right)$ from each k-point in the reciprocal cell in the direction to the nearest neighbors.

For the summation in Eq. (10) we have proceeded in four steps: In the first step the second-order dipole moments $M_{\alpha, \alpha_{1} \alpha_{2}}^{\kappa_{1} \kappa_{2}}(\mathbf{q})$ of Eq. (2) are evaluated for $\mathbf{q}$ vectors on a 


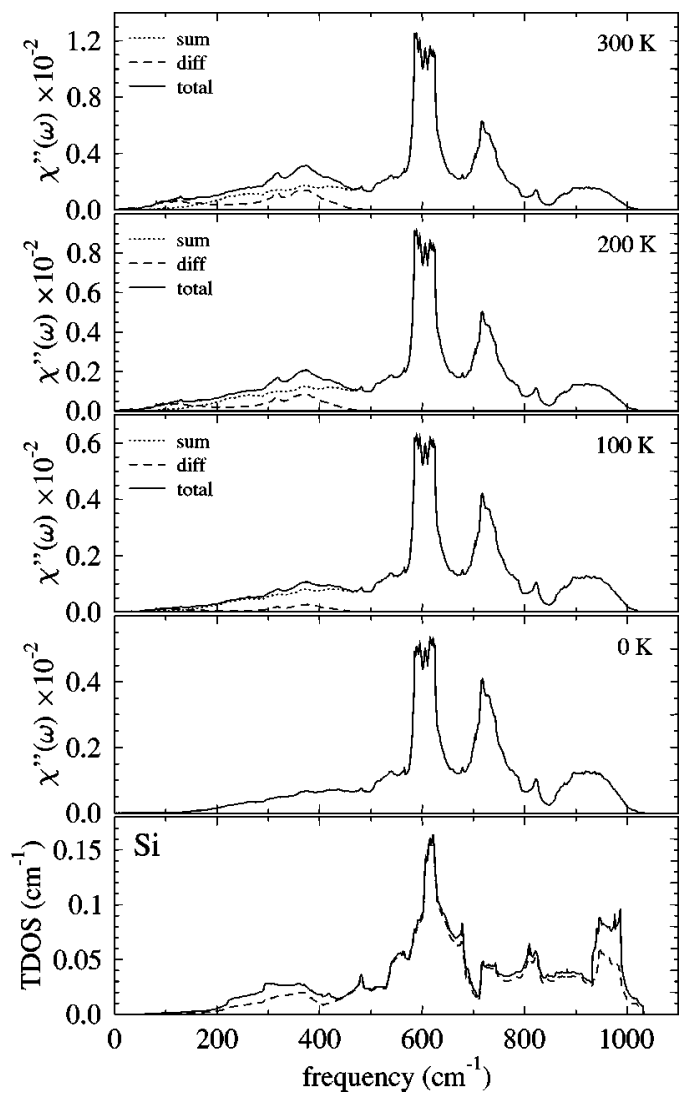

FIG. 1. Si. TDOS with (full line) and without (dashed line) overtones at zero temperature and imaginary part of the dielectric susceptibility $\chi$ of silicon due to two-phonon processes. The temperatures are from top to bottom $300,200,100$, and $0 \mathrm{~K}$. The dotted lines illustrate the contribution from the summation processes, the dashed lines those from the difference processes, and the full lines the total dielectric function. Note the different scales.

$4 \times 4 \times 4$ q-vector mesh. We have checked our results for high symmetry points against results which we have obtained from frozen-phonon-type methods. The second step is the Fourier transformation to the real-space coefficients, which are determined completely up to the eighth neighbors according to the inverse transformation of the one given in Eq. (2). The third step is the Fourier transformation like in Eq. (2) of the real-space second-order dipole-moments to reciprocal space on a denser mesh of $\mathbf{q}$ vectors. ${ }^{27}$ In a final step, the summation over the wave vector $\mathbf{q}$ in Eq. (10) is performed by the tetrahedron method. ${ }^{28,29}$

\section{RESULTS}

At zero temperature the dielectric susceptibility is determined by just the summation processes, since the contribution of the difference processes vanishes due to the factor $n_{\lambda_{2}}-n_{\lambda_{1}}$ in Eq. (10). If the matrix elements $M_{\alpha}^{(2)}\left(\mathbf{q} j_{1}\right.$, $-\mathbf{q} j_{2}$ ) were independent of $\mathbf{q}$ and $j$ the susceptibility would be proportional to $D_{2}^{+}(\omega)$ of Eq. (11), which is shown in the bottom panel of Figs. 1 and 2 for of silicon and germanium, respectively. The summation processes have a cut-off at twice the maximum frequency, $2 \omega_{\max }, \omega_{\max }$ being the Ra-

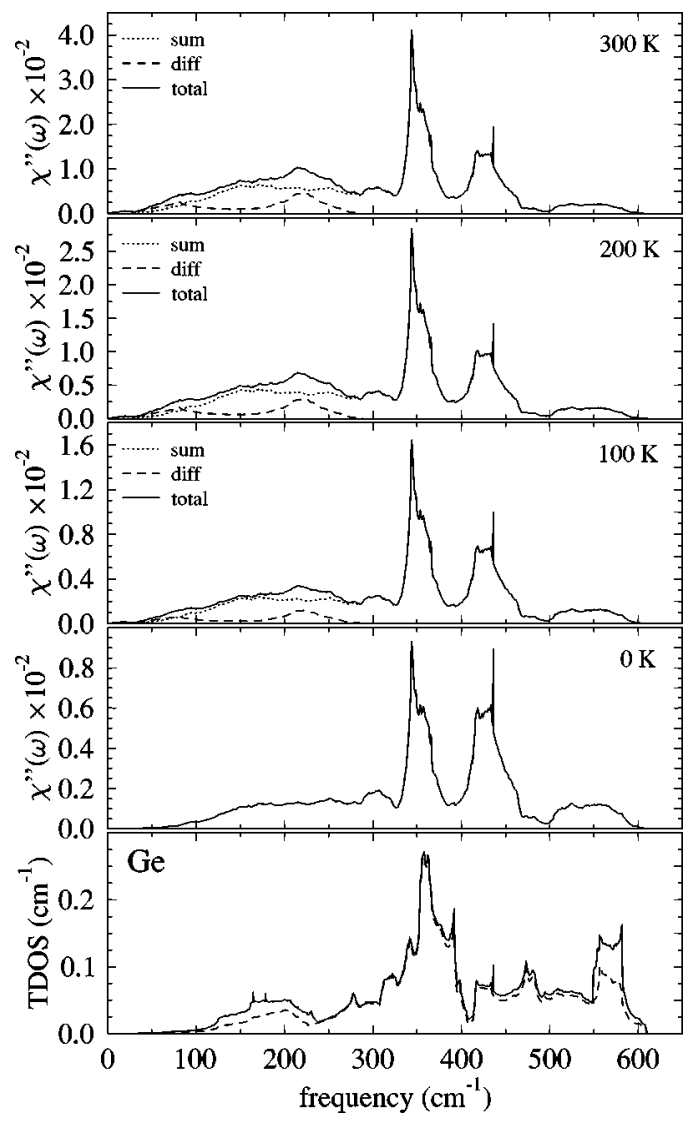

FIG. 2. Same as Fig. 1 but for Ge.

man frequency. The close similarity of the phonon dispersion curves of $\mathrm{Si}$ and $\mathrm{Ge}$ is reflected in the similarity of the $D_{2}^{+}(\omega)$ spectra.

Since the overtones, i.e., those with $j_{1}=j_{2}$, do not contribute to the IR spectra we show the TDOS with and without these contributions; the most obvious difference occurs in the (2LO) overtone region of the rather dispersionless uppermost dispersion sheet of predominantly longitudinal optical (LO) modes. A wealth of so-called critical points (spikes and kinks) can be noticed, some of which are due to overtones, for example that near $300 \mathrm{~cm}^{-1}$ for $\mathrm{Si}^{30}$

In addition to cutting out the contributions of the overtones the influence of the matrix elements $M_{\alpha, \alpha_{1} \alpha_{2}}^{\kappa_{1} \kappa_{2}}(\mathbf{q})$ is to give different weights to the various combinations (with $j_{1}$ $\left.\neq j_{2}\right)$ as is illustrated by the difference between the two bottom panels of each figure. A number of critical points are strongly suppressed or even disappear.

Within lowest-order perturbation theory, the temperature dependence of the susceptibility spectra is due to the Bose functions, see Eq. (12). With increasing temperature (up to $200 \mathrm{~K})$ the contribution of the difference processes increases much more strongly than that of the summation processes. At higher temperature, e.g., at $300 \mathrm{~K}$ and above, the contributions of both processes are of comparable magnitude in the one-phonon regime. (The cut-off energy of the difference processes is at $\omega_{\max }$.) At high temperatures the contributions of both processes are linearly proportional to the temperature. 


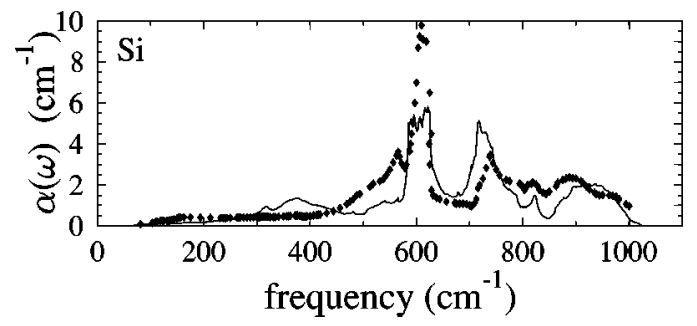

FIG. 3. Absorption coefficient of silicon due to two-phonon processes at $293 \mathrm{~K}$. The experimental data (diamonds) are taken from Ref. 31.

From the susceptibility we have calculated the absorption coefficient of silicon at a temperature of $293 \mathrm{~K}$. The shape as well as the intensity are in overall good agreement with the experimental spectrum of Ikezawa and Ishigame; ${ }^{31}$ see Fig. 3. The spectral maxima at approximately 600, 720, and $810 \mathrm{~cm}^{-1}$ are reproduced. The theoretical intensity of the maximum at $600 \mathrm{~cm}^{-1}$ is smaller than the experimental one, while the one at $720 \mathrm{~cm}^{-1}$ is larger. In the low-frequency range there is a peak at $390 \mathrm{~cm}^{-1}$ which is not seen in the experiment.

The analogous results for germanium are compared with the experimental data of Ikezawa and $\mathrm{Nanba}^{32}$ in Fig. 4. We find excellent agreement of the peak positions at 350 and $430 \mathrm{~cm}^{-1}$. A striking difference can be seen at the shoulder (near $270 \mathrm{~cm}^{-1}$ ) of the large maximum, which appears in the experiment but not the theoretical spectrum.

\section{DISCUSSION}

Numerical inaccuracies may have entered the calculation, since the second-order dipole moments of Eq. (7) turn out to be the difference of large numbers. However, since effective charges calculated within the LDA differ only very little from the experimental values, we believe the same to be true for their changes with atomic displacements, i.e., for the second-order dipole moments.

Also, the difference between theoretical and experimental spectra may be due to (interference with) higher-order processes, since the experimental lower-frequency band intensities depend on temperature non-linearly at room temperature and somewhat below. ${ }^{31,32}$ Whether or not the experimental data contain contributions from higher than second order could only be decided from a more detailed investigation of the temperature dependence of the spectra.

The main dependence of the spectra on temperature comes from the explicit temperature dependence of the Bose factors. In principle, the phonon eigen solutions, too, enter-

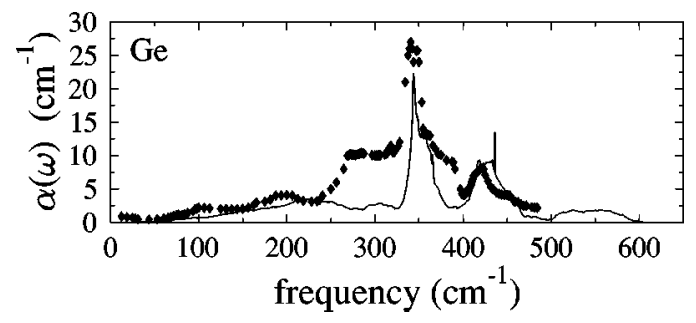

FIG. 4. Absorption coefficient of germanium due to two-phonon processes at $293 \mathrm{~K}$. The experimental data (diamonds) are taken from Ref. 32.

ing expressions like Eq. (9) depend upon temperature due to anharmonic renormalization. Since the anharmonicity of the phonons in $\mathrm{Si}$ and $\mathrm{Ge}$ is small ${ }^{5,6,10}$ the temperature dependent anharmonic shift and width can give only a small correction to the spectra, which is far less important than the influence of the Bose functions. This is supported by the fact that the theoretical peak positions agree well with the experimental ones, namely the IR as well as in the neutron spectra.

Despite the differences, our calculations show an overall satisfying agreement with the experimental spectra. Not only the general shape but in particular the overall intensity is well reproduced without introducing any experimental input. This is the main progress as compared to the calculation of Ref. 14, where a larger number of phenomenological parameters is needed to reproduce the spectra with deviations from the experimental data similar to or larger than the present results. Also, the results of Ref. 15, even though from first principles at the beginning, contained a number of arbitrary parameters at the end. It turns out that the second-order dipole moments from neighbors beyond the second shell have noticeable contributions to the spectra in the upper frequency regime.

\section{SUMMARY}

Summarizing we have presented a theory in the framework of DFPT for the calculation of the second-order dipole moments. The numerical application to the two-phonon infrared absorption of $\mathrm{Si}$ and Ge has led to a reasonably good agreement of the theoretical and experimental spectra without the introduction of any experimental input. The discrepancies must remain unexplained for the time being.

\section{ACKNOWLEDGMENT}

We would like to thank the Deutsche Forschungsgemeinschaft (Contract No. STR 118/24) for financial support of this work.
${ }^{1}$ P. Giannozzi, S. de Gironcoli, P. Pavone, and S. Baroni, Phys. Rev. B 43, 7231 (1991).

${ }^{2}$ S. Baroni, S. de Gironcoli, A. Dal Corso, and P. Giannozzi, Rev. Mod. Phys. 73, 515 (2001).

${ }^{3}$ X. Gonze and J.P. Vigneron, Phys. Rev. B 39, 13120 (1989).

${ }^{4}$ A. Debernardi and S. Baroni, Solid State Commun. 91, 813
(1994).

${ }^{5}$ A. Debernardi, S. Baroni, and E. Molinari, Phys. Rev. Lett. 75, 1819 (1995).

${ }^{6}$ G. Lang, K. Karch, M. Schmitt, P. Pavone, A.P. Mayer, R.K. Wehner, and D. Strauch, Phys. Rev. B 59, 6182 (1999).

${ }^{7}$ A. Debernardi, Phys. Rev. B 57, 12847 (1998). 
${ }^{8}$ A. Debernardi, Solid State Commun. 113, 1 (2000).

${ }^{9}$ A. Debernardi, C. Ulrich, K. Syassen, and M. Cardona, Phys. Rev. B 59, 6774 (1999).

${ }^{10}$ G. Deinzer, G. Birner, and D. Strauch, Phys. Rev. B 67, 144304 (2003).

${ }^{11}$ A. Dal Corso and F. Mauri, Phys. Rev. B 50, 5756 (1994).

${ }^{12}$ A. Dal Corso, F. Mauri, and A. Rubio, Phys. Rev. B 53, 15638 (1996).

${ }^{13}$ G. Deinzer and D. Strauch, Phys. Rev. B 66, 100301 (2002).

${ }^{14}$ W. Kress, H. Borik, and R.K. Wehner, Phys. Solid State 29, 133 (1968).

${ }^{15}$ D. Strauch, W. Windl, H. Sterner, P. Pavone, and K. Karch, Physica B 219 \& 220, 442 (1996).

${ }^{16}$ D. Strauch, P. Pavone, A.P. Mayer, K. Karch, H. Sterner, A. Schmid, Th. Pletl, R. Bauer, and M. Schmitt, in Festkörperprobleme/Advances in Solid State Physics, edited by R. Helbig (Vieweg, Braunschweig/Wiesbaden, 1998), Vol. 37, p. 99.

${ }^{17}$ R.D. King-Smith and D. Vanderbilt, Phys. Rev. B 47, 1651 (1993).

${ }^{18}$ R. Resta, Rev. Mod. Phys. 66, 899 (1994).

${ }^{19}$ E.I. Blount, in Solid State Physics, edited by F. Seitz and D. Turnbull (Academic, New York, 1962), Vol. 13, p. 305.

${ }^{20}$ H. Bilz, D. Strauch, and R.K. Wehner, in Handbuch der Physik,
Vol. XXV/2d: Licht und Materie, edited by S. Flügge (Springer, Berlin, 1984).

${ }^{21}$ U. von Barth and R. Car (unpublished).

${ }^{22}$ D.M. Ceperley and B.J. Alder, Phys. Rev. Lett. 45, 566 (1980).

${ }^{23}$ J.P. Perdew and A. Zunger, Phys. Rev. B 23, 5048 (1981).

${ }^{24}$ H.J. Monkhorst and J.D. Pack, Phys. Rev. B 13, 5188 (1976).

${ }^{25}$ N. Marzari and D. Vanderbilt, Phys. Rev. B 56, 12847 (1997).

${ }^{26}$ In contrast to the evaluation of the polarisation via the Berry phase, we do not have a phase ambiguity by taking wave functions from different $\mathbf{k}$ points or even additional $\mathbf{k}$ points, because all phases are entering Eq. (7) together with their complex conjugate.

${ }^{27}$ In Ref. 15 the even coarser $\mathbf{q}$-vector mesh had been incomplete, and a Fourier interpolation was, therefore, not possible. Realspace coefficients had been optimized to reproduce the q-space coefficients, and the coefficients for the fine mesh of $\mathbf{q}$-vectors, calculated from the real-space coefficients, contained inherent errors.

${ }^{28}$ G. Lehmann and M. Taut, Phys. Status Solidi B 54, 469 (1972).

${ }^{29}$ G. Gilat and N.R. Bharatiya, Phys. Rev. B 12, 3479 (1975).

${ }^{30}$ The spike near $430 \mathrm{~cm}^{-1}$ in the theoretical spectra of Ge is a numerical artifact.

${ }^{31}$ M. Ikezawa and M. Ishigame, J. Phys. Soc. Jpn. 50, 3734 (1981).

${ }^{32}$ M. Ikezawa and M. Nanba, J. Phys. Soc. Jpn. 45, 148 (1978). 